\title{
Comparison of the effects of post-spinning drawing and wet stretching on regenerated silk fibers produced through straining flow spinning
}

\author{
Rodrigo Madurga ， Alfonso M. Gañán-Calvo , Gustavo R. Plaza , \\ José Miguel Atienza ，Gustavo V. Guinea ， Manuel Elices . Patricia A. López ， \\ Rafael Daza , Daniel González-Nieto ， José Pérez-Rigueiro
}

\begin{abstract}
A B S T R A C T
Straining Flow Spinning is a versatile and robust spinning technique for the production of regenerated silkworm silk fibers using mild chemistries. However, reaching high values of tensile strength and strain at breaking requires a step of wet-stretching in water, which limits scalability and the practical usage of the technique. Here, we show that adding a post-spinning drawing step to the procedure improves the performance of the fibers, and allows the development of a scalable process. It is also shown that the properties of the fiber can be tuned by varying the parameters of the post-spinning step. Finally, equivalence is established between the discrete wet-stretching process and the continuous postspinning drawing step.
\end{abstract}

\section{Introduction}

The interest in spider and silkworm silks originates from the combination of high values of strain at breaking and tensile strength characteristic of the natural materials [1], and from their biocompatibility [2]. However, applications of the natural silks, as well as of their bioinspired counterparts beyond the textile industry is hampered by the difficulties of producing fibers at an industrial scale without chemical treatments that damage the material and in a cost effective way. In an attempt to overcome these difficulties, different procedures were proposed for the production of high-performance silk-based fibers since the turn of the Century.

The different spinning processes share the common principle of producing a solid fiber from a silk-protein solution, and differ in the mechanism that induces the assembly of the proteins. In this context, wet-spinning was and still is one of the preferred solutions. In a wet-spinning process the dope enters into a coagulating bath that removes the solvent and leads to the formation of the fiber [3-5]. Simple variations of wet-spinning are offered by dry spinning [6], in which the solvent of the dope evaporates to the atmosphere, and dry jet wet-spinning $[7,8]$, in which the solution goes through a small air gap before entering the coagulating bath.

However, an increasing knowledge of the principles of natural spinning has thrust the development on more sophisticated processes that try to exploit the chemical and physical processes found in Nature [9]. These processes comprise the existence of a $\mathrm{pH}$ gradient $[10,11]$, an ion gradient $\left(\mathrm{Na}^{+}, \mathrm{K}^{+}, \mathrm{Ca}^{2+}\right.$ and $\left.\mathrm{Cl}^{-}\right)$[12] or a gradient of protein concentration [13,14] along the spinning gland. These mechanisms are thought to favor the organization of the solubilized proteins either as micelles [15], as liquid crystals [16,17] or as a combination of both [16], so that the transition from soluble (dope) to solid (fiber) conformation can be performed in a 


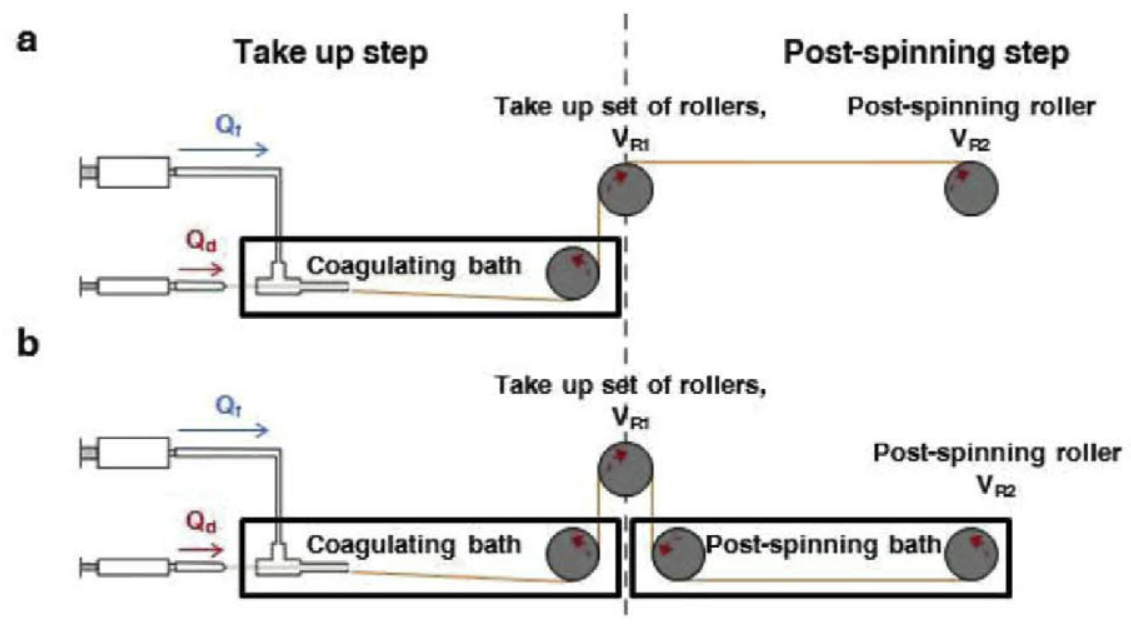

Fig. 1. Scheme of the post-spinning drawing step (a) in air and (b) in water coupled to the SFS process.

controlled way. Beside the relevant role assigned to the chemistry of the process, mechanical stresses are also thought to play a critical role in the transition of the protein structure to the solid conformation during the natural process $[1,18]$.

This combined knowledge on the chemistry and physics of the natural spinning process has led to several biomimetic microfluidic devices for the production of artificial silk [19,20]. In this context, our group has developed the Straining Flow Spinning (SFS) technique, which has been shown to be a versatile and robust biomimetic spinning procedure [21] that can produce artificial silk fibers under a wide range of mild chemical conditions [22]. However, high performance fibers, i.e. with a work to fracture that approaches that of natural material $\left(\sim 60 \mathrm{MJ} / \mathrm{m}^{3}\right)$ were invariably obtained after a wetstretching process. This process implies stretching the fiber in water, fixing the deformed length, removing water and allowing the fiber to dry. Although the process is easily implemented at a laboratory scale, it would imply a difficult two-step process engineering design to convert it into an industrially scalable process.

In this work we show that an equivalent effect to the discrete wet-stretching process is obtained by adding a post-spinning drawing step during spinning. Post-spinning drawing consists of a continuous stretching of the fiber either in water or in air immediately after the fiber is solidified and retrieved by a take-up roller. In contrast to the wet-stretching process, post-spinning drawing can be easily implemented as a one-step process at an industrial level, which opens the possibility of using the highperformance SFS fibers for numerous applications, including the biomedical field.

\section{Materials and methods}

\subsection{Preparation of the dope}

The details of the preparation of the dope can be found elsewhere [22]. Briefly, silkworm (Bombyx mori) silk cocoons were degummed and subsequently solved to a concentration of $10 \%(w /$ v) in an $8 \mathrm{M} \mathrm{LiBr}$ and $0.1 \mathrm{M} \mathrm{NH}_{4} \mathrm{HCO}_{3}$. Solving was followed by a dialysis step against deionized water. Finally, the solutions were concentrated by reverse dialysis. The final composition of the dope was fibroin $16 \%(\mathrm{w} / \mathrm{v})$ in $1 \mathrm{M} \mathrm{CaCl}_{2}$ aqueous solution.

\subsection{Spinning parameters}

The basic details of the SFS process can be found elsewhere [21].
A scheme of the technique is shown in Fig. 1, including the experimental setup with the post-spinning drawing module. The geometrical parameters used were: diameter of the orifice of the nozzle, $D_{1}=400 \mu \mathrm{m}$, inner diameter of the capillary, $d_{1}=150 \mu \mathrm{m}$, tapering angle at the end of the capillary, $\alpha=90^{\circ}$ and distance between the take-up and the post-spinning drawing rollers, $\mathrm{L}_{\mathrm{R}}=60 \mathrm{~cm}$.

The coagulating bath and the focusing fluid had the same composition consisting of a mixture of ethanol and an aqueous acetic acid $1 \mathrm{M}$ solution in a ratio 80:20 [22].

The three hydrodynamic parameters of the take-up step were kept constant: flow rate of the dope, $Q_{d}=5 \mu \mathrm{l} / \mathrm{min}$, flow rate of the focusing fluid, $Q_{\mathrm{f}}=0.4 \mathrm{ml} / \mathrm{min}$, and velocity of the take up roller, $V_{R 1}=3.5 \mathrm{~m} / \mathrm{min}$. The velocity of the post-spinning roller, $V_{R 2}$, was varied depending on the process, although it was usually fixed to the maximum speed that did not lead to the breaking of the fiber. The post-spinning draw ratio is defined as $D R_{P S}=V_{R 2} / V_{R 1}$.

Post-spinning drawing was performed either in air or in water. The different combinations of the spinning parameters tested in this work are summarized in Table 1 .

\subsection{Characterization of the regenerated fibers}

The details of the mechanical and microstructural characterization of the regenerated samples are presented elsewhere $[21,23,24]$. Briefly, samples were mounted on aluminium foil frames with a base length of $10 \mathrm{~mm}$ and tested in an Instron 4411 tensile testing machine. At least three samples for each condition were tensile tested. Forces were measured with a precision balance (Precisa XT220A, resolution $0.1 \mathrm{mg}$ ). The initial length of the fiber, $\mathrm{L}_{0}$, was measured with a caliper and the diameter of the fibers was measured with an optical microscope (Leica DMI 3000B) using a $40 \mathrm{X}$ objective. Tensile tests were performed at a constant speed of $1 \mathrm{~mm} / \mathrm{min}$ and either in air (nominal environmental conditions:

Table 1

Spinning conditions tested in the present study.

\begin{tabular}{lllll}
\hline Code & Post-spinning media & $V_{R 1}$ & $V_{R 2}$ & DRPS \\
\hline NoPS & & $3.5 \mathrm{~m} / \mathrm{min}$ & - & 0 \\
PSA1.8 & Air & $3.5 \mathrm{~m} / \mathrm{min}$ & $6.4 \mathrm{~m} / \mathrm{min}$ & 1.8 \\
PSA3.6 & Air & $3.5 \mathrm{~m} / \mathrm{min}$ & $12.7 \mathrm{~m} / \mathrm{min}$ & 3.6 \\
PSW1.8 & Water & $3.5 \mathrm{~m} / \mathrm{min}$ & $6.4 \mathrm{~m} / \mathrm{min}$ & 1.8 \\
PSW3.6 & Water & $3.5 \mathrm{~m} / \mathrm{min}$ & $12.7 \mathrm{~m} / \mathrm{min}$ & 3.6 \\
\hline
\end{tabular}


$\mathrm{T}=25^{\circ} \mathrm{C}$ and $\mathrm{RH}=35 \%$ ) or immersed in water at $20^{\circ} \mathrm{C}$. Stresses were calculated using the diameter measured from the optical micrographs assuming a circular cross-sectional area.

The hypothesis that the volume of the fiber remains constant upon stretching [25] was used for calculating true stresses. True stress and true strain were calculated as:

$\varepsilon=\operatorname{Ln} \frac{L}{L_{0}}$

$\sigma=\frac{F}{A}=F \frac{L}{A_{0} L_{0}}$

where $A_{0}$ and $L_{0}$ are the initial area and length of the sample, respectively, and $A$ and $L$ are the instantaneous values of these magnitudes.

The microstructure of regenerated silkworm silk fibers was analyzed by infrared spectroscopy using a Nicolet iS5 spectrometer equipped with an iD5 ATR complement. IR spectra were obtained from a bundle of fibers that were folded into a small ball, and pressed against the diamond window at constant pressure. All spectra were the result of averaging 64 measurements in the range from 550 to $4000 \mathrm{~cm}^{-1}$ with a resolution of $4 \mathrm{~cm}^{-1}$. The amide I peak of each spectrum was then fitted with Gaussians [26]. Details of the procedure used for the assignment of the peaks is presented as Supplementary Material.

The possible presence of calcium in the regenerated fibers was assessed through Energy Dispersive X-ray Spectroscopy (EDS) using an X Flash Detector 5010 (Bruker) coupled to a Zeiss Auriga FESEM. A representative spectrum of an as spun NoPS fiber is presented as Supplementary Data.

\subsection{Wet-stretching process, supercontraction and recovery test}

Wet stretching is a simple procedure for controlling and enhancing the mechanical performance of silk fibers developed by the authors and used with natural spider silk [27] and regenerated silkworm silk fibers [28]. It consists of stretching a fiber immersed in water up to a selected strain, fixing its ends, and allowing it to dry overnight. In this work samples were wet-stretched up to a value of $92 \%$ of their strain at breaking in water.

To test if the regenerated fibers showed supercontraction, fibers were allowed to contract in water and then allowed to dry overnight. Supercontraction was quantified by the percentage of supercontraction \%SC as:

$\% S C=\frac{L_{0}-L_{M S}}{L_{0}} \times 100$

Where $L_{M S}$ is the length of the fiber after supercontraction.

Recovery tests were performed to assess the existence of a true supercontracted state in regenerated silk fibers. In a recovery test, the fiber is stretched in air up to a given strain. The deformed fiber is then allowed to contract freely in water and to dry overnight. Subsequently, the fiber is stretched again in air up to a similar or higher value of strain. The stretching-contraction steps can be repeated several times at different strains until the strain at breaking is reached. Concurrence of the curves subjected to recovery tests confirms the existence of a ground state to which the fiber can revert independently of its loading history [29].

\section{Results and discussion}

A scheme of the straining flow spinning device, including the take-up and post-spinning drawing step modules is shown in Fig. 1.
In all cases continuous spinning for more than $1 \mathrm{~h}$ was possible for every spinning condition, which implies a length of fiber up to $800 \mathrm{~m}$ for the fastest spinning condition (PSA3.6 and PSW3.6).

Individual fibers were retrieved and mounted for the tensile tests. Fig. 2a shows representative true stress-true strain curves of the as-spun fibers tested in air. It can be appreciated that the fibers spun without post-spinning (black curve) show a brittle behavior, while all the fibers spun with a post-spinning step show a more ductile behavior with values of strain at breaking ranging from 0.05 to 0.20 and reaching a maximum value of the work to fracture of up to $21 \pm 4 \mathrm{MJ} / \mathrm{m}^{3}$ (average \pm standard error) for PSW1.8 samples.

When the fibers were tested in water the true stress-true strain curves (Fig. 2b) changed drastically and showed properties that resemble those of an elastomer. Although the post-spinning drawing step did not lead to significant changes in the tensile strength, an apparent tendency to decreasing strain at breaking with increasing DRPS is observed. It is worth mentioning that all samples contracted when immersed in water, except those spun with no post-spinning (NoPS). The values of the percentage of supercontraction, as obtained from equation (3), were $11.6 \pm 0.8 \%$ and $11 \pm 1 \%$ for the fibers post-spun in air with $\mathrm{DRPS}_{\mathrm{PS}}=1.8$ and 3.6. respectively; and $16.5 \pm 0.5 \%$ and $17.0 \pm 0.5 \%$ for the fibers postspun in water with DRPS $=1.8$ and 3.6, respectively. These results indicate a possible dependence of the percentage of supercontraction on the medium in which post-spinning drawing is performed. In contrast, the draw ratio does not seem to exert any significant influence on the percentage of supercontraction.

The tensile properties of maximum supercontracted fibers, i.e. fibers allowed to supercontract in water and then allowed to dry, are shown in Fig. 2c. Fibers subjected to post-spinning drawing show an increase in their strain at breaking after supercontraction with no significant change in their tensile strengths. On the other hand, fibers without post-spinning (i.e. NoPS with $\mathrm{DR}_{P S}=0$ ) do not show any change in their mechanical properties upon immersion in water and subsequent drying. In the maximum supercontracted state, the post-spun fibers reach values of the work to fracture of up to $1 / 3$ (PSA1.8: $W_{\mathrm{f}}=19 \pm 3$, PSA3.6: $W_{\mathrm{f}}=24 \pm 5$ and PSW1.8: $W_{\mathrm{f}}=23 \pm 4 \mathrm{MJ} / \mathrm{m}^{3}$ ) or up to half (PSW3.6: $\mathrm{W}_{\mathrm{f}}=34 \pm 4 \mathrm{MJ} / \mathrm{m}^{3}$ ) of the work to fracture of natural silkworm silk $\left(\mathrm{W}_{\mathrm{f}}=60 \mathrm{MJ} / \mathrm{m}^{3}[30]\right)$. The tensile properties of a supercontracted PSW3.6 fiber and those of a representative degummed natural silkworm silk fiber $[21,23,24]$ are compared in Fig. $2 d$.

Although the shrinkage of the fiber in an aqueous environment is an indicator of supercontraction, its confirmation requires establishing the existence of a ground state to which the fiber can revert independently from its previous loading history. The presence of this ground state is indicated by the concurrence of the stress-strain curves after contraction, and assessed through recovery tests [29]. Three samples of each post-spinning condition (NoPS samples were not analyzed, since they do not contract in water), were subjected to recovery tests, i.e. to cycles of stretching in air and contraction in water. The results of the recovery tests are shown in Fig. 3 (a: PSA1.8, b: PSA3.6, c: PSW1.8, d: PSW3.6). In a first cycle the fibers were stretched up to a strain of $10 \%$ (black curves) followed by supercontraction. In a second cycle the fibers were stretched up to a strain of $20 \%$ (red curves) followed by a second supercontraction step. Finally, the fibers were stretched up to breaking in a third cycle (blue curves). In all cases true strain was calculated using the initial length of the fiber, $\mathrm{L}_{\mathrm{MS}}$, after the initial supercontraction step and, consequently, before the first loading cycle. This way a displacement of the curve to the right in subsequent cycles indicates that the fiber does not recover its initial length completely. It can be observed that the fibers PSA1.8 and PSW1.8 do not recover their initial length after being stretched to $20 \%$ of strain (Fig. $3 \mathrm{a}$ and c), whereas the fibers PSA3.6 and PSW3.6 

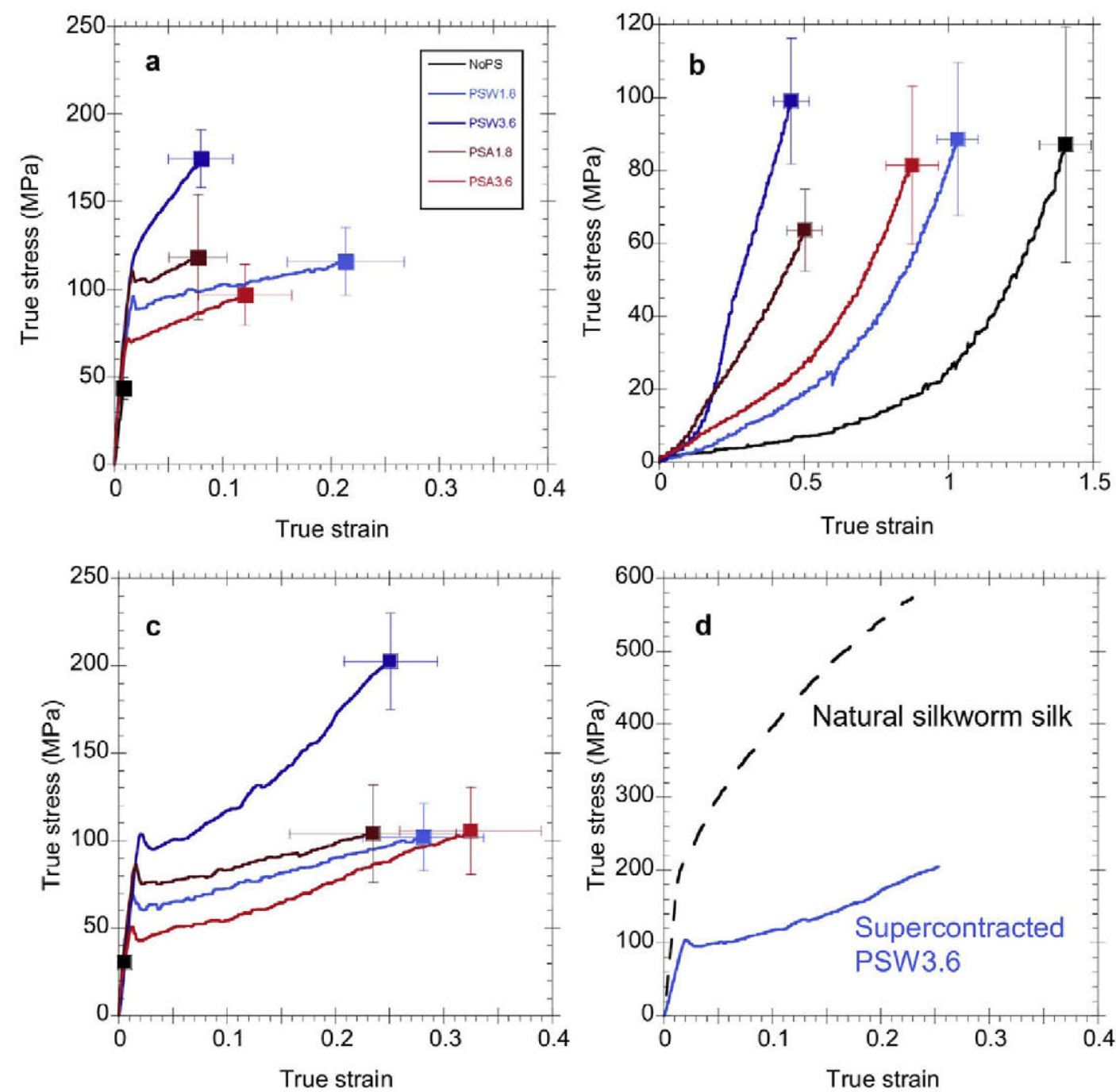

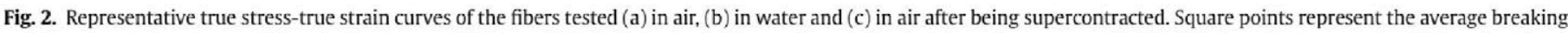

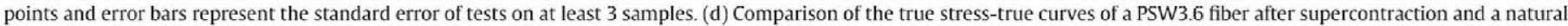
silkworm silk fiber after degumming $[21,23,24]$.

always recover their initial length (Fig. 3 b and d).

FTIR-ATR spectroscopy was used to complement the data obtained from the mechanical tests in an attempt to study the influence of post-spinning drawing on the microstructure of the fibers. Fig. 4 shows the amide I peak of fibers produced with different post-spinning treatments. The amide I peak of degummed natural silkworm silk (gray curve) is also shown to allow comparison. Although the spectra of the regenerated fibers show an increase in the noise in the $1640-1680 \mathrm{~cm}^{-1}$ region, which corresponds to random coil and helical conformations, no significant differences can be established between the various regenerated fibers. This conclusion is supported by the deconvolution of the amide I peaks in their elementary contributions (see Supplementary Data).

The possible presence of calcium from the dope in the regenerated fibers was assessed by Energy Dispersive X-ray Spectroscopy (EDS, see details in Supplemetary Data). No calcium peak was detected up to the resolution limit of the technique, that can be roughly estimated as $0.5 \%$ of the total number of atoms of the sample following the specifications of the manufacturer.

In previous works on regenerated silk fibers it was observed that stretching the fibers in water-in a process known as wet-stretchingcan switch their mechanical properties from brittle to ductile [21].
Since a comparable behavior is found in SFS fibers subjected to post-spinning drawing, it is worth exploring whether wet stretching and post-spinning drawing represent two alternative possibilities of tuning the mechanical behavior of the fibers.

In order to compare the effects of wet stretching and postspinning drawing, five fibers spun from each set of spinning conditions were subjected to a wet-stretching process up to $92 \%$ of their strain at breaking in water. As it is shown in Fig. 5a, all wetstretched samples show concurring true stress-true strain curves, including comparable values of strain at breaking, after being allowed to supercontract. Consequently, it can be concluded that, independently of the initial as-spun state of the fibers, wetstretching allows reaching a common final state for the fibers produced under the spinning conditions given above.

The true stress-true strain curves of the wet-stretched samples and those of the maximum supercontracted PSW3.6 samples are compared in Fig. 5b. The concurrence of all the curves indicates the equivalent effect of post-spinning drawing and wet-stretching on the regenerated fibers, and supports the idea that a common molecular mechanism underlies both procedures. In this regard, it is illustrative that PSW3.6 samples correspond to the maximum speed of the post-spinning roller that did not lead to the breaking of 

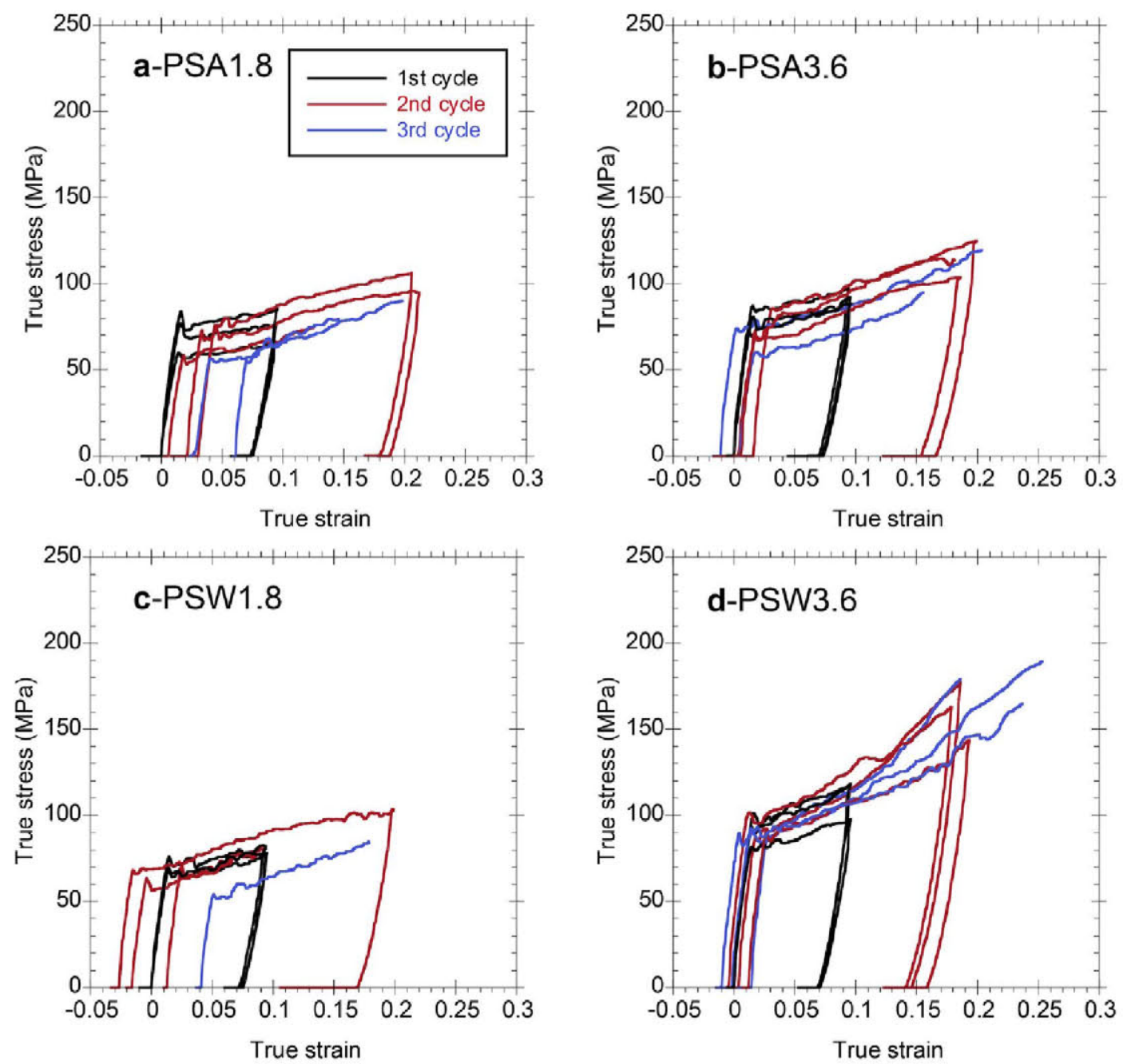

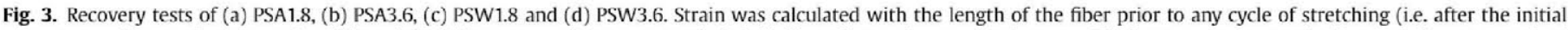

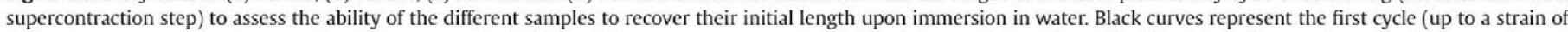

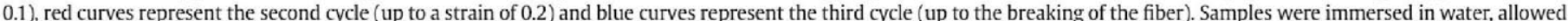
to supercontract and dried between cycles. (For interpretation of the references to colour in this figure legend, the reader is referred to the Web version of this article.)

the fiber.

Interestingly, the effect exerted by water on the tensile behaviour of SFS regenerated fibers contrasts with that observed in natural silkworm silk, and is comparable to that found in major ampullate gland (MAS) spider silk. This parallelism suggests that the effect of post-spinning drawing on SFS fibers might be described with a single parameter, as occurs with the alignment parameter, $\alpha^{*}$, in the MAS fibers of Entelegynae spiders [31]. An alignment parameter, $\alpha$, can be defined for the SFS fibers from the initial length of the fiber, $\mathrm{L}_{\mathrm{i}}$, and the length of the fiber subjected to post-spinning drawing and subsequent supercontraction, $\mathrm{L}_{\mathrm{f}}$, as:

$\alpha=\operatorname{Ln} \frac{L_{f}}{L_{i}}=\operatorname{Ln}\left((1-\% S C / 100) \cdot D R_{P S}\right)$

The definition of $\alpha$ takes into account that the length of the fiber, LPs, after being subjected to a post-spinning drawing of draw ratio, DRPs, is:

$L_{P S}=L_{i} \cdot D R_{P S}$

under the assumption that the elastic deformation during the postspinning drawing step is negligible compared to the irreversible deformation. If the fiber is then allowed to supercontract in water, the final length of the fiber, $\mathrm{L}_{\mathrm{f}}$, will be

$L_{f}=(1-\% S C / 100) L_{P S}$

Equation (4) is the result of combining equations (5) and (6) in the original definition of the parameter $\alpha$.

In Fig. 6 the true strain-true stress curves of the fibers tested in water with different values of DRps previously shown in Fig. $2 \mathrm{~b}$ have been displaced along the true strain axis a value corresponding to the alignment parameter, $\alpha$, calculated from equation (4). Except for the fibers PSA3.6, all fibers are shown to concur at large values of true strain, as previously described for MAS spider silk [31].

PSA3.6 samples (dark red curves) are singular, since their true stress-true strain curves do not concur with the others if displaced a value of $\alpha$ along the true strain axis. This anomalous behavior can be explained by the possible sliding of the fiber on the take-up roller due to the stresses induced by the post-spinning step. The sliding effect does not seem to occur in the PSW3.6 samples because the take-up roller configuration for post-spinning in water is composed of a set of three rollers instead of two, as it is used for post-spinning drawing in air (see Fig. 1). In addition, sliding is likely to increase for processes with higher values of DRPs. The sliding of 


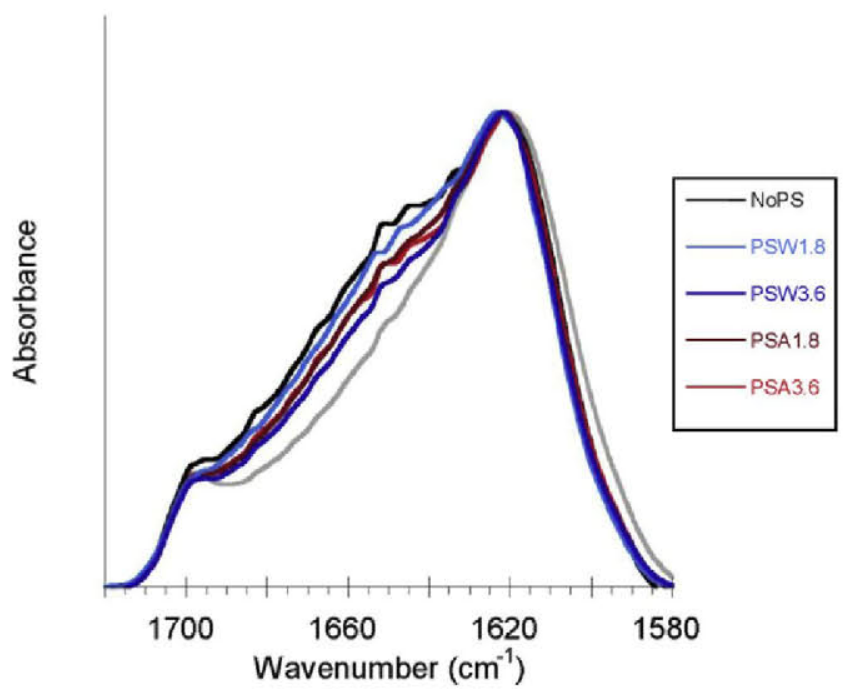

Fig. 4. FTIR microstructural analysis of the fibers spun under different spinning conditions. (a) Representative Amide I peaks of the different spinning conditions in comparison to natural silkworm silk fibers (gray). Samples are identified as: NoPSblack, PSA1.8-light red, PSA3.6-dark red, PSW1.8-light blue and PSW3.6-dark blue.

the fiber on the take up roller implies that the actual value of DRPS to which the fiber is subjected is lower than that corresponding to the nominal value of the draw ratio. As a matter of fact, it is possible to displace the curve along the true strain axis in Fig. 6 (broken line), so that the PSA3.6 curve also concurs with the other curves at high values of true strain. The concurrence of all curves upon being displaced in accordance with the value of the alignment parameter indicates that all fibers share a common state, which manifests itself at high values of strain.

\section{Conclusions}

In this work it is demonstrated that the addition of a postspinning drawing step to the SFS process is equivalent to subjecting the regenerated fibers to wet-stretching. Consequently, wet stretching and post-spinning drawing are found to represent two possibilities of materializing a single molecular mechanism that

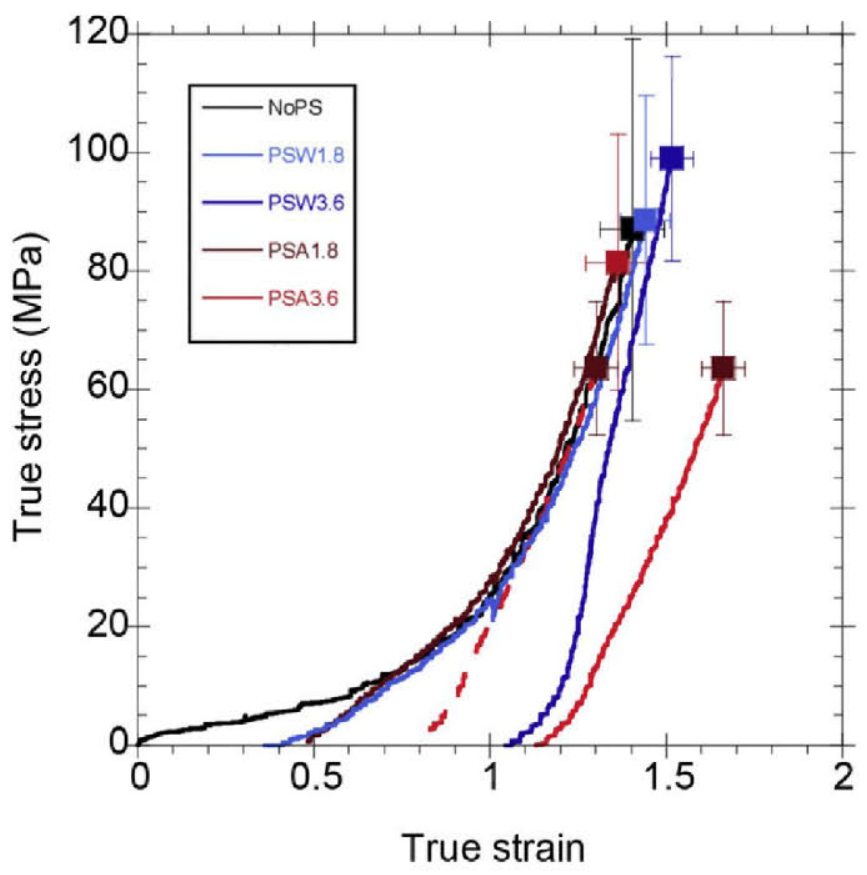

Fig. 6. Concurrence at high values of strain of the true stress-true strain curves of fiber tested in water upon being displaced along the true strain axis a value equal to $\alpha$ (see definition of the alignment parameter, $\alpha_{2}$ in the text). Spinning conditions are identified as: NoPS-black, PSA1.8-light red, PSA3.6-dark red, PSW1.8-light blue and PSW3.6-dark blue. Squared points represent the average breaking points and error bars represent the standard error of tests on at least 3 samples. (For interpretation of the references to colour in this figure legend, the reader is referred to the Web version of this article.)

allows tuning the mechanical behavior of the fibers. It is also shown that both procedures lead to the production of high performance regenerated silk fibers.

However, and in spite of their fundamental equivalence, major practical differences are apparent between both procedures. In contrast to wet-stretching, post-spinning drawing can be incorporated to the spinning process and applied continuously to the fiber, which simplifies its scaling up to an industrial level considerably.

Additionally, regenerated fibers subjected to either a post-
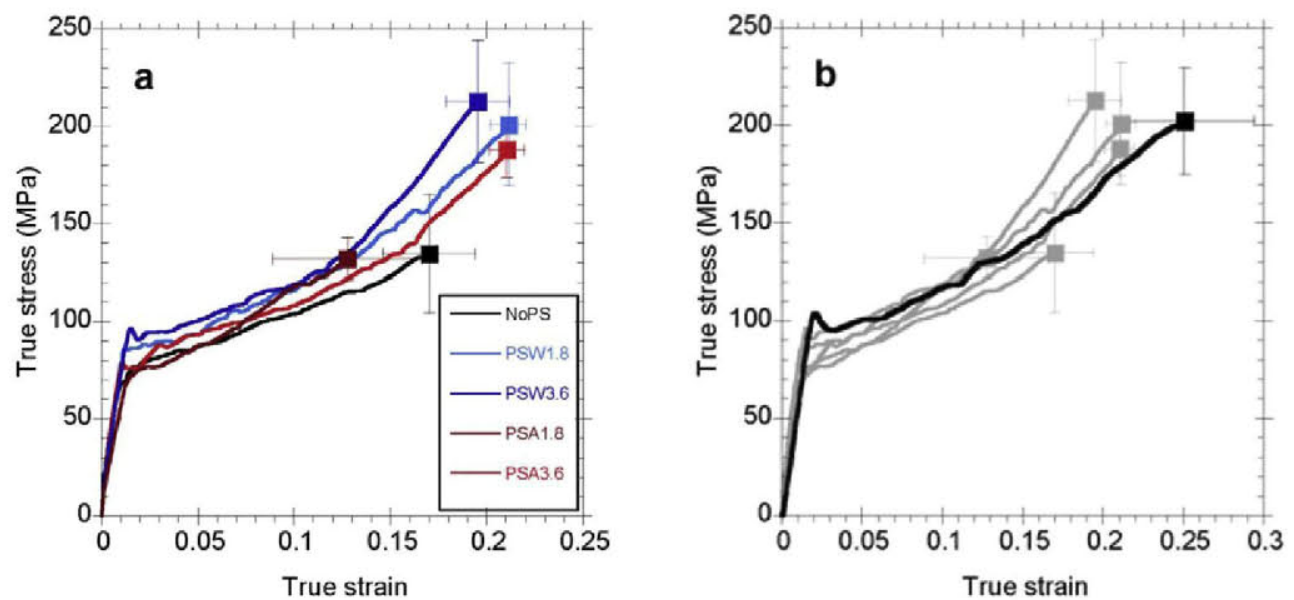

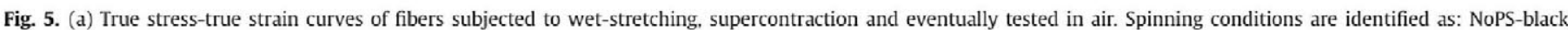

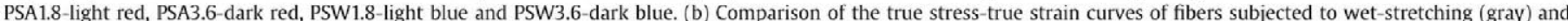

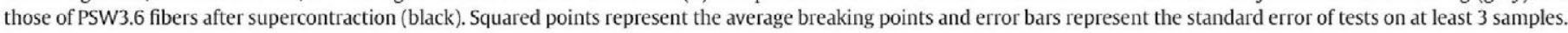
(For interpretation of the references to colour in this figure legend, the reader is referred to the Web version of this article.) 
spinning drawing step or to wet-stretching may show supercontraction, a feature characteristic of spider major ampullate gland silk, which implies the existence of a ground state to which the fibers can revert independently of their previous loading histories. The parallelism between SFS regenerated fibers and spider silk is extended by the definition of an alignment parameter, $\alpha$, in the former which determines the tensile behaviour of any given fiber in terms of the processing conditions.

\section{Author contributions}

R.M. performed the experimental work, except for the EDS analysis that was performed by P.A.L., R.D. and D.G.-N. G.V.G, M.E., G.R.P. and J.M.A. contributed to the data analysis and reviewed the manuscript. J.P.-R. and A.M.G.-C. proposed the experiments. The main text was written by R.M. and J.P.-R.

\section{Conflicts of interest}

Patent application of the Straining Flow Technology (SFS) was filed on December 18th, 2015.

\section{Acknowledgements}

Straining Flow Spinning was developed at the Universidad Politécnica de Madrid and Universidad de Sevilla. Cocoons were kindly provided by Prof. José Luis Cenis (IMIDA, Spain). The work was funded by Ministry of Economy and Competitiveness in Spain through Projects MAT2016-75544-C2-1-R, MAT2016-79832-R, DPI2013-46485-C3-1-R and DPI2016-78887-C3-1-R.

\section{Appendix A. Supplementary data}

Supplementary data related to this article can be found at https://doi.org/10.1016/j.polymer.2018.07.042.

\section{References}

[1] M. Heim, D. Keerl, T. Scheibel, Spider silk: from soluble protein to extraordi nary fiber, Angew Chem. Int. Ed. Engl. 48 (2009) 3584-3596.

[2] C. Vepari, D. Kaplan, Silk as a biomaterial, Prog. Polym. Sci. 32 (2007) 991-1007.

[3] O. Liivak, A. Blye, N. Shah, L.W. Jelinski, A microfabricated wet-spinning apparatus to spin fibers of silk proteins. Structure-property correlations, Macromolecules 31 (1998) 2947-2951.

[4] 1.C. Um, C.S. Ki, H.Y. Kweon, K.G. Lee, D.W. Ihm, Y.H. Park, Wet spinning of silk polymer - II. Effect of drawing on the structural characteristics and properties of filament, Int. J. Biol. Macromol. 34 (2004) 107-119, https://doi.org/10.1016 j.ijbiomac.2004.03.011 ER.

[5] F. Zhang, Q. Lu, X. Yue, B. Zuo, M. Qin, F. Li, D.L. Kaplan, X. Zhang, Regeneration of high-quality silk fibroin fiber by wet spinning from $\mathrm{CaCl} 2$-formic acid solvent, Acta Biomater. 12 (2015) 139-145, https://doi.org/10.1016 j.actbio.2014.09.045.

[6] X. Yue, F. Zhang, H. Wu, J. Ming, Z. Fan, B. Zuo, A novel route to prepare dryspun silk fibers from $\mathrm{CaCl} 2$-formic acid solution, Mater. Lett. 128 (2014) 175-178, https://doi.org/10.1016/j.matlet.2014.04.116.

[7] P. Corsini, J. Perez-Rigueiro, G.V. Guinea, G.R. Plaza, M. Elices, E. Marsano, M.M. Carnasciali, G. Freddi, Influence of the draw ratio on the tensile and fracture behavior of NMMO regenerated silk fibers, J. Polym. Sci. B Polym. Phys. 45 (2007) 2568-2579, https://doi.org/10.1002/polb.21255 ER.

[8] G.R. Plaza, P. Corsini, E. Marsano, J. Perez-Rigueiro, M. Elices, C. Riekel, C. Vendrely, G.V. Guinea, Correlation between processing conditions, microstructure and mechanical behavior in regenerated silkworm silk fibers,
J. Polym. Sci. B Polym. Phys. 50 (2012) 455-465, https://doi.org/10.1002/ polb.23025.

[9] S. Yazawa, Spinning of concentrated aqueous silk fibroin solution, J. Chem. Soc. Japan 63 (1960) 1428-1430.

[10] L.J. Domigan, M. Andersson, K.A. Alberti, M. Chesler, Q. Xu, J. Johansson, A. Rising, D.L. Kaplan, Carbonic anhydrase generates a $\mathrm{pH}$ gradient in Bombyx mori silk glands, Insect Biochem. Mol. Biol. 65 (2015) 100-106, https:// doi.org/10.1016/j.ibmb.2015.09.001.

[11] M. Andersson, J. Johansson, A. Rising, Silk spinning in silkworms and spiders, Int. J. Mol. Sci. 17 (2016) 1290, https://doi.org/10.3390/ijms17081290. UNSP 1290.

[12] C.W.P. Foo, E. Bini, J. Hensman, D.P. Knight, R.V. Lewis, D.L. Kaplan, Role of pH and charge on silk protein assembly in insects and spiders, Appl. Phys. Mater. Sci. Process 82 (2006) 223-233, https://doi.org/10.1007/s00339-005-3426-7 ER.

[13] X Chen, D.P. Knight, F. Vollrath, Rheological characterization of Nephila spidroin solution, Biomacromolecules 3 (2002) $644-648$, https://doi.org/ $10.1021 / \mathrm{bm} 0156126$

[14] D.H. Hijirida, K.G. Do, C. Michal, S. Wong, D. Zax, L.W. Jelinski, C-13 NMR of Nephila clavipes major ampullate silk gland, Biophys. I. 71 (1996) 3442-3447.

[15] H.J. Jin, D.L. Kaplan, Mechanism of silk processing in insects and spiders, Nature 424 (2003) 1057-1061, https://doi.org/10.1038/nature01809 ER.

[16] A. Rising, J. Johansson, Toward spinning artificial spider silk, Nat. Chem. Biol. 11 (2015) 309-315, https://doi.org/10.1038/NCHEMBIO.1789.

[17] F. Vollrath, D.P. Knight, Liquid crystalline spinning of spider silk, Nature 410 (2001) 541-548

[18] J. Magoshi, Y. Magoshi, M.A. Becker, S. Nakamura, Biospinning by Bombyx mori Silkworm, Abstracts of Papers of the American Chemical Society, 1996, p. 212,53 .

[19] R. Konwarh, P. Gupta, B.B. Mandal, Silk-microfluidics for advanced biotechnological applications: a progressive review, Biotechnol. Adv. 34 (2016) 845-858, https://doi.org/10.1016/j.biotechadv.2016.05.001.

[20] Q. Peng, Y. Zhang, L. Lu, H. Shao, K. Qin, X. Hu, X. Xia, Recombinant spider silk from aqueous solutions via a bio-inspired microfluidic chip, Sci. Rep. 6 (2016) 36473, https://doi.org/10.1038/srep36473.

[21] R. Madurga, A.M. Ganan-Calvo, G.R. Plaza, G.V. Guinea, M. Elices, J. PerezRigueiro, Production of high performance bioinspired silk fibers by straining flow spinning, Biomacromolecules 18 (2017) 1127-1133, https://doi.org/ 10.1021/acs.biomac.6b01757.

[22] R. Madurga, A.M. Ganan-Calvo, G.R. Plaza, G.V. Guinea, M. Elices, J. PerezRigueiro, Straining flow spinning: production of regenerated silk fibers under a wide range of mild coagulating chemistries, Green Chem. 19 (2017) 3380-3389, https://doi.org/10.1039/c7gc01254c.

[23] J. Perez-Rigueiro, C. Viney, J. Llorca, M. Elices, Silkworm silk as an engineering material, J. Appl. Polym. Sci. 70 (1998) 2439-2447.

[24] J. Perez-Rigueiro, C. Viney, J. Llorca, M. Elices, Mechanical properties of silkworm silk in liquid media, Polymer 41 (2000) 8433-8439.

[25] G.V. Guinea, J. Perez-Rigueiro, G.R. Plaza, M. Elices, Volume constancy during stretching of spider silk, Biomacromolecules 7 (2006) 2173-2177, https:// doi.org/10.1021/bm060138v ER.

[26] X. Hu, D. Kaplan, P. Cebe, Determining beta-sheet crystallinity in fibrous proteins by thermal analysis and infrared spectroscopy. Macromolecules 39 (2006) 6161-6170, https://doi.org/10.1021/ma0610109.

[27] G.V. Guinea, M. Elices, J. Perez-Rigueiro, G.R. Plaza, Stretching of supercontracted fibers: a link between spinning and the variability of spider silk, J. Exp. Biol. 208 (2005) 25-30.

[28] G.R. Plaza, P. Corsini, J. Perez-Rigueiro, E. Marsano, G.V. Guinea, M. Elices, Effect of water on Bombyx mori regenerated silk fibers and its application in modifying their mechanical properties, J. Appl. Polym. Sci. 109 (2008) 1793-1801, https://doi.org/10.1002/app.28288.

[29] M. Elices, J. Perez-Rigueiro, G. Plaza, G.V. Guinea, Recovery in spider silk fibers, J. Appl. Polym. Sci. 92 (2004) 3537-3541, https://doi.org/10.1002/app.20383 ER.

[30] G. Belen Perea, C. Solanas, N. Mari-Buye, R. Madurga, F. Agullo-Rueda, A. Muinelo, C. Riekel, M. Burghammer, I. Jorge, J. Vazquez, G.R. Plaza, A.L. Torres, F. del Pozo, G.V. Guinea, M. Elices, J. Luis Cenis, J. Perez-Rigueiro, The apparent variability of silkworm (Bombyx mori) silk and its relationship with degumming, Eur. Polym. J. 78 (2016) 129-140, https://doi.org/10.1016/ j.eurpolymj.2016.03.012.

[31] R. Madurga, G.R. Plaza, T.A. Blackledge, G.V. Guinea, M. Elices, J. PerezRigueiro, Material properties of evolutionary diverse spider silks described by variation in a single structural parameter, Sci. Rep. 6 (2016) 18991, https:// doi.org/10.1038/srep18991. 